\title{
Correction: Comparison of Canadian public medication insurance plans and the impact on out-of-pocket costs
}

An earlier version of this article (published Nov. 27, 2017) contained errors in Figures 1 and 2, and Table 1. ${ }^{1}$ The data were incorrect for New Brunswick.

The errors have been corrected at cmajopen.ca.

\section{Reference}

1. Campbell DJT, Manns BJ, Soril LJJ, et al. Comparison of Canadian public medication insurance plans and the impact on out-of-pocket costs. CMAf
Open 2017;5:E808-13.

CMAJ Open 2018. DOI:10.9778/cmajo.20170167 